\title{
New Knowledge and Evidence for Better Leadership
}

Greta Cummings, RN, PhD

CIHR New Investigator

AHFMR Population Health Investigator

Professor, Faculty of Nursing,

University of Alberta

Editor, Canadian Journal of Nursing Leadership

The Canadian healthcare system is increasingly complex and more difficult to manage with each passing decade - a situation compounded by near-constant change in health authority structures, interprofessional teamwork and conflict, the actions and decisions of senior administrators and government officials, changing public demographics and consumer expectations of the healthcare system, and the implementation of new knowledge, evidence and innovation. Through these changes - or despite them - nurses remain the largest group of healthcare providers at the interface of the healthcare system and the patient/client. Nurses must continue working with individuals and families to improve health outcomes while managing through systems changes and translating them into everyday work settings for themselves and their clients.

Hence the need for nursing leadership to guide the transformation of today's reality into a preferred future for the nursing profession as a whole, for each nursing role and each practice setting. We need to know how we will collaborate interprofessionally, and how we - the largest healthcare profession - will shape the system and ultimately improve the health of Canadians.

We often say that we need leadership. But what kind of leadership does the nursing profession need? How can health outcomes for patients in hospitals, clients at home and in communities, and residents of long-term care facilities be improved? What are the mechanisms by which leadership practices by nurses at any level of the system influence better health and system outcomes? How can we develop the kind of leadership that we need? To address these and many more questions, we need research to develop new knowledge and build on existing knowledge. 
My passion in research is leadership in nursing and the healthcare system, and my mission is to investigate the ways in which various leadership styles or approaches influence the healthcare workforce and health outcomes, both positively and negatively. The important relationships that nursing leadership styles have with outcomes for nursing work environments, the nursing workforce and for patients are being clearly documented in the research literature. A recent systematic literature review of 63 research reports on such relationships found evidence of highly differential effects of relational leadership styles (focused on people and relationships) compared to task-focused leadership styles (focused on job completion, deadlines and directives). Relational leadership styles were associated with key outcomes such as significantly higher nurse job satisfaction, organizational commitment, staff satisfaction with work, role and pay, staff relationships with work, staff health and well-being, work environment factors, and productivity and effectiveness. Task-focused leadership styles were associated with significantly lower values of all these outcomes. Other studies have shown that relational leadership styles of hospital nursing administrators also influence important patient outcomes, including rates of hospital-acquired infections, adverse events and 30-day patient mortality. Leadership styles focused on task completion alone are clearly insufficient to achieve optimum outcomes in the healthcare system.

Nursing leadership research is not particularly amenable to laboratory settings or the restricted controlled approaches of randomized controlled trials, but is best based in the real-life complex care settings where leadership occurs, decisions are made, policy is enacted and individuals provide and receive care. This consideration presents challenges to the research teams who are interested in leadership research, but also provides opportunities for research collaborations between nursing researchers, decision-makers, educators and nurses in a variety of clinical roles. Many such successful research collaborations have been led by researchers across the country - Alice Gaudine (Memorial University of Newfoundland), Norma J. Murphy (Dalhousie University), Dr. Judith Ritchie (McGill University Health Centre), Heather Laschinger and Carol Wong (University of Western Ontario) and Maura MacPhee (University of British Columbia), to name just a few.

Many more researchers and senior nurse leaders in health authorities have developed new knowledge and evidence to guide leadership practice. Some of these topics include authentic leadership, coaching, communications, creativity innovation, decision-making, education leadership, emotional intelligence, ethics, governance, high-performance teams, knowledge management, leading change, retention strategies, partnerships, performance, individual and team development, program evaluation, research methodology, relationships, strategic planning, vision and mission. 
Translating knowledge into practice in our complex health system is one of the biggest leadership challenges that we face. Studies such as TREC: Translating Research in Elder Care (Carole Estabrooks, principal investigator, University of Alberta) and TROPIC: Translating Research on Pain in Children (Bonnie Stevens, principal investigator, University of Toronto/SickKids Hospital) are two examples of effects to examine the influence of the context within which nurses work (including leadership, culture, evaluation and resources) on their ability to implement new evidence and change health outcomes for seniors in long-term care facilities (TREC) and children undergoing painful procedures in hospital (TROPIC).

Over the next year, CJNL will profile studies that examine the effectiveness of interventions to improve leadership, and to transform practice, in any of the topics above. Across the country in most Canadian healthcare facilities, nursing administrators are leading initiatives to improve the quality of their nurses' work environments, and the health outcomes of individuals and families. Measuring the impact in particular contexts is challenging, yet necessary. We welcome manuscripts that address any of these topics. We also welcome new reviewers who can assist us in ensuring that all articles are relevant, timely and robust in their contributions. If you are interested in reviewing for CJNL, please contact Dianne FosterKent, managing editor at dkent@longwoods.com.

I look forward to working with Lynn Nagel, editor-in-chief, and my co-editors, Mike Villaneuve (policy) and Patricia Petryshen (practice). 